\title{
Evaluación teórica de la capacidad de procesos de Rational Unified Process respecto del MoProSoft
}

${ }^{(1)}$ Katia Canepa Vega

(2)Abraham Dávila

\section{RESUMEN}

La determinación de la capacidad de los procesos en empresas que desarrollan software es un tema que ha cobrado interés, pues a través de ellas se las puede calificar en un determinado nivel de capacidad o madurez, según el modelo usado. Además, algunas empresas cuentan con un conjunto de prácticas y metodologías de trabajo establecidas, que en el caso del Perú es frecuente que se basen en Rational Unified Process. En este trabajo, se presenta la evaluación teórica de las capacidades de los procesos en la categoría de operación del modelo de procesos de software MoProSoft bajo el supuesto que una organización tenga implementado de manera adecuada un conjunto mínimo de elementos de RUP.

Palabras clave: RUP, ISO/IEC 15504, MoProSoft.

TheORETICAL EVALUaTION OF THE ABILITY TO process on the Rational Unified Process MoProsoftx

\section{ABSTRACT}

Determining the processes capabilities in companies that develop software is an issue that has gained interest because through them they can be classified at a certain level of capability or maturity, depending on the model used. In addition, some companies have a set of established practices and working methods, in the case of Peru is often based on Rational Unified Process (RUP). This paper presents the theoretical evaluation of the processes capabilities in the operation category of software processes model Moprosoft under the assumption that an organization has properly implemented a set of minimal elements of RUP.

Keywords: XXXXXXXXXXXXXXXXXXXXXXXXX

\section{INTRODUCCIÓN}

La calidad del proceso software es un tema que interesa cada vez más a la industria de software por los beneficios que les puedan ofrecer; algunas empresas lo hacen por las mejoras en la manera de hacer las cosas y otras por los logros obtenibles como son las certificaciones de procesos. Dentro de los modelos existentes en el mercado, uno de los más representativo es el Capability and Maturity Model Integrated (CMMI) [Heinz 2003] desarrollado por el Software Engineering Institute; pero existen otros como: la ISO/IEC 90003 que es la guía de la implementación de la ISO 9001:2000 [ISO 2004] de carácter internacional, el modelo denominado Melhoria de Procesos de Software (MPS) de Brasil [SOFTEX 2007]; y el Modelo de Procesos de Software (MoProSoft) de México [Oktaba 2005], entre otros. Estos modelos establecen referentes que las empresas deben alcanzar para incrementar las capacidades de sus procesos o madurez organizacional a través de procesos de mejora continua. En esta misma dirección, se desarrolló el proyecto COMPETISOFT, que es un esfuerzo de investigadores y profesionales Iberoamericanos que buscan incrementar la competitividad de las pymes desarrolladoras de software utilizando un marco común de modelos que han sido pensados para su realidad [COMPETISOFT 2006], [Oktaba 2007]. COMPETISOFT se basa en el modelo de referencia de procesos mexicano MoProSoft y se han realizando diversas implementaciones en la región [Mogrovejo 2008]; [Sánchez 2008]; [Oktaba 2007]; [Astorga 2006].

De lo que se conoce en la industria de software en el Perú, se sabe que una cantidad significativa de empresas y universidades utilizan Rational Unified Process (RUP), un marco de trabajo para definir metodologías de desarrollo de software. Tomando en cuenta esta situación, se inició el trabajo de análisis teórico entre los modelo de MoProSoft y RUP, dentro del marco del proyecto COMPETISOFT en donde participa el Grupo de Investigación y Desarrollo de Ingeniería de Software (GIDIS) de la Pontificia Universidad Católica del Perú (PUCP). El primer resultado de este trabajo fue la determinación de la cobertura de RUP respecto de MoProSoft en los procesos de la categoría de operaciones [Canepa 2008]. El segundo resultado es la determinación teórica de la capacidad de procesos de una empresa que tiene

1 Egresada de Ingeniería de Sistemas de la UNMSM.

E-mail: katia.canepa@gmail.com

2 Magíster en Informática, PUCP, Profesor del Departamento de Ingeniería de la PUCP.

E-mail: abraham.davila@pucp.edu.pe 
implementado un conjunto de elementos de RUP y que será evaluado con el modelo MoProSoft, en los procesos de la categoría de operaciones. Este segundo resultado cobra importancia pues permitirá a las empresas que tengan implementado RUP ver la posibilidad de evaluar sus procesos usando MoProSoft y tener un cierto grado de confianza que si están siguiendo adecuadamente las definiciones de RUP, podría alcanzar determinado nivel de capacidad en MoProSoft. El mapeo se realiza a nivel de operaciones debido a que MoProSoft cubre temas de gestión de las empresas que no es el alcance de RUP.

En este artículo se presenta la determinación de la capacidad teórica respecto de los procesos de la categoría de operaciones de una organización que tiene implementado de manera adecuada (y es usado) un conjunto determinado de elementos de RUP. El artículo está organizado de la siguiente manera: la sección 2 presenta el modelo MoProSoft y el marco de trabajo Rational Unified Process, la sección 3 presenta la capacidad de procesos, la sección 4 presenta el cálculo de la capacidad de los procesos, la sección 5 presenta las discusiones de los resultados y la sección 6 las conclusiones y trabajos futuros.

\section{MODELO MOPROSOFT Y RATIONAL UNIFIED PROCESS}

En esta sección se describe brevemente el Modelo de Procesos de Software (MoProSoft) y el Rational Unified Process (RUP) con el propósito de explicar cómo se realizó el mapeo entre ambos y que constituye la base para calcular la capacidad de los procesos considerados.

\section{MoProSoft}

MoProSoft se desarrolló a partir de un conjunto de requisitos de la industria de software mexicano, que está principalmente constituido por pymes [Oktaba 2005]. MoProSoft es un modelo basado en procesos e incorpora aspectos de negocios y buenas prácticas de la ingeniería de software ajustadas para el tamaño de las pymes. Desde el año 2004, MoProsoft ha sido probado en empresas mexicanas [Oktaba 2005a] y en el 2005, fue adoptado como Norma Técnica Mexicana [Oktaba 2005].
MoProSoft ha sido tomado como base del proyecto COMPETISOFT [Oktaba 2007] y también ha sido tomado como base para la definición de perfiles en el proyecto internacional "Very Small Enterprises" del WG-24 de la ISO/IEC JTC1/SC7 [Laporte 2008]. Adicionalmente, MoProSoft ha sido adoptado como Norma Técnica Peruana NTP-291.100 desde mayo del 2009 [Dávila 2009].

MoProSoft propone una estructura de tres niveles, los cuales están en correspondencia con las categorías básicas organizacionales: Alta Dirección, Gestión y Operación [Oktaba 2005]. La Figura 1 muestra estas categorías y la distribución de los procesos a través de ellas, así como los subprocesos de la Gestión de Recursos. Todos los procesos se presentan a través de un patrón de procesos tal como se muestra en la Figura 2.

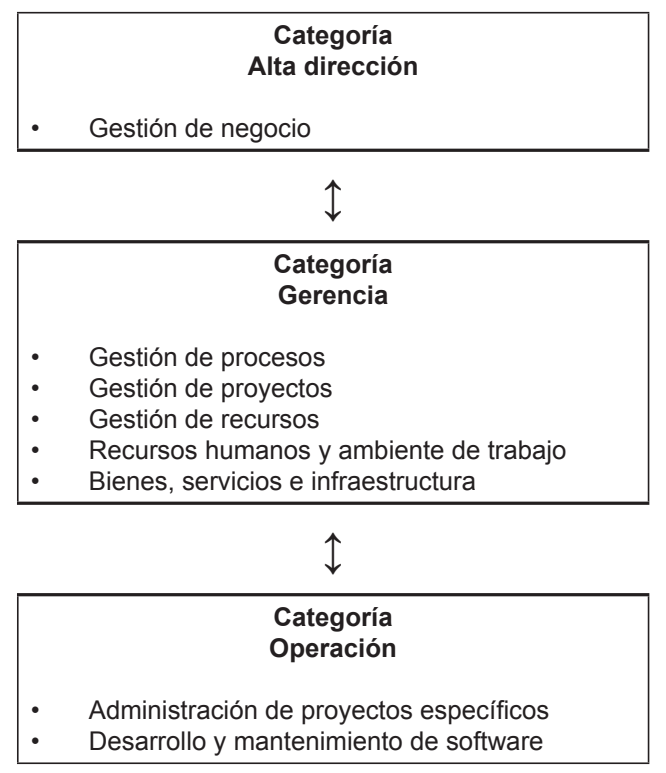

Fuente: Tomado de MoProSoft [Oktaba 2005].

Figura 1. Categorías y procesos de MoProSoft.

El patrón tiene tres secciones: definición general del proceso, prácticas y guías de ajuste. La guía de MoProSoft utiliza el patrón para documentar todos los procesos y lo recomienda en caso la organización no cuente con uno [Oktaba 2005]. 


\begin{tabular}{|ll|}
\hline \multicolumn{1}{|c|}{ Patrón de proceso } \\
\hline Descripción general del proceso \\
\hline - & Proceso \\
- & Categoría \\
- & Propósito \\
- & Descripción \\
- & Indicadivos \\
- & Metas cuantitativas \\
- & Responsabilidad y autoridad \\
- & Subprocesos (opcional) \\
- & Procesos relacionados \\
- & Entradas. Fuentes de entradas \\
- & Prodas. Destino de salida \\
- & Referencias blibliográficas \\
\hline Prácticas \\
\hline - & Roles involucrados, nombre, abreviatura, capacitación \\
- & Actividad, mapeo con objetivos, tareas, roles en tareas \\
- & Diagrama de flujo de trabajo \\
- & Verificación y validación, Identificación, actividad, pro- \\
\hline & ducto, rol, descripción \\
\hline - & Incorporación de infraestructura, actividad, recurso. \\
- & Capacitación \\
\hline - & Suía de ajuste \\
\hline - & Lecciones aprendidas \\
\hline & Identificación de la guía \\
\hline
\end{tabular}

Fuente: Adaptado de MoProSoft [Oktaba 2005].

Figura 2. Patrón de Procesos de MoProSoft.

Una definición de procesos contiene por lo menos un conjunto de actividades, las relaciones existentes, los criterios que indican el inicio fin del proceso, así como roles, aplicaciones y datos de las actividades por lo que el patrón de MoProSoft cubre ampliamente esta definición de procesos [ISO 2004a].

\section{Rational Unified Process}

Rational Unified Process (RUP) es un marco de referencia de procesos que incorpora las mejores prácticas de la industria de desarrollo de software [Rational 1998]. RUP es definido a través de tres principales conceptos: RUP como un proceso de ingeniería de software cuyo pilar principal es el establecimiento de un proceso iterativo e incremental, guiado por casos de uso y centrado en la arquitectura [Jacobson 1999], considerado como un producto, integrado con el conjunto (suite) de herramientas de IBM Rational y como un marco de referencia de procesos, adaptado o extendido a las necesidades de la organización [Kruchten 2003].

La estructura de RUP está basado en dos dimensiones: una dimensión está basada en el tiempo que presenta cuatro fases, que muestran la organización temporal del proyecto que a su vez pueden ser divididas en iteraciones; y una segunda dimensión, basada en los contenidos que define nueve disciplinas organizadas según actividades [IBM 2007]. Adicionalmente, muestra el esfuerzo que debe realizarse por cada disciplina en cada fase, el mismo que varía en función al tiempo. En la Figura 3 se muestra esta estructura.

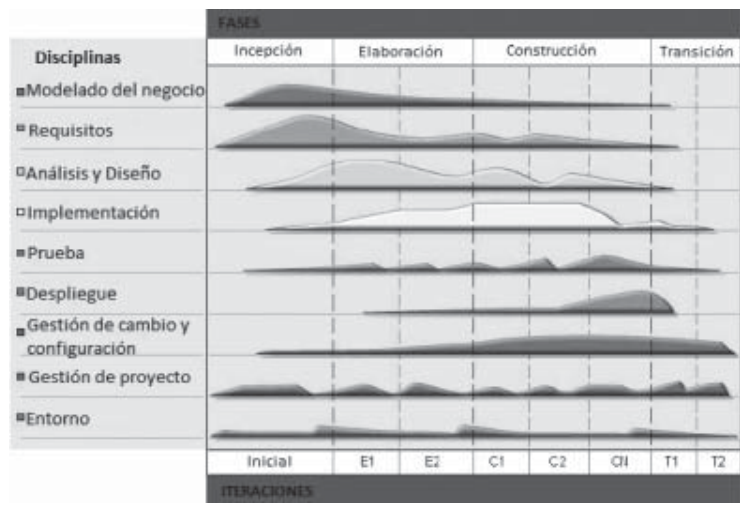

Fuente: Adaptado de RUP [IBM 2007].

Figura 3. Estructura de RUP.

\section{CAPACIDAD DE LOS PROCESOS}

La capacidad de un proceso se define, según la ISO/IEC 15504, como un conjunto de atributos que le da la habilidad a un proceso para conseguir los objetivos de negocios actuales o futuros [ISO 2004c]. La evaluación se basa en la valoración de los atributos de procesos con lo que se puede determinar el nivel de capacidad de dicho proceso. La Figura 4 muestra los elementos del método de evaluación y sus relaciones. 


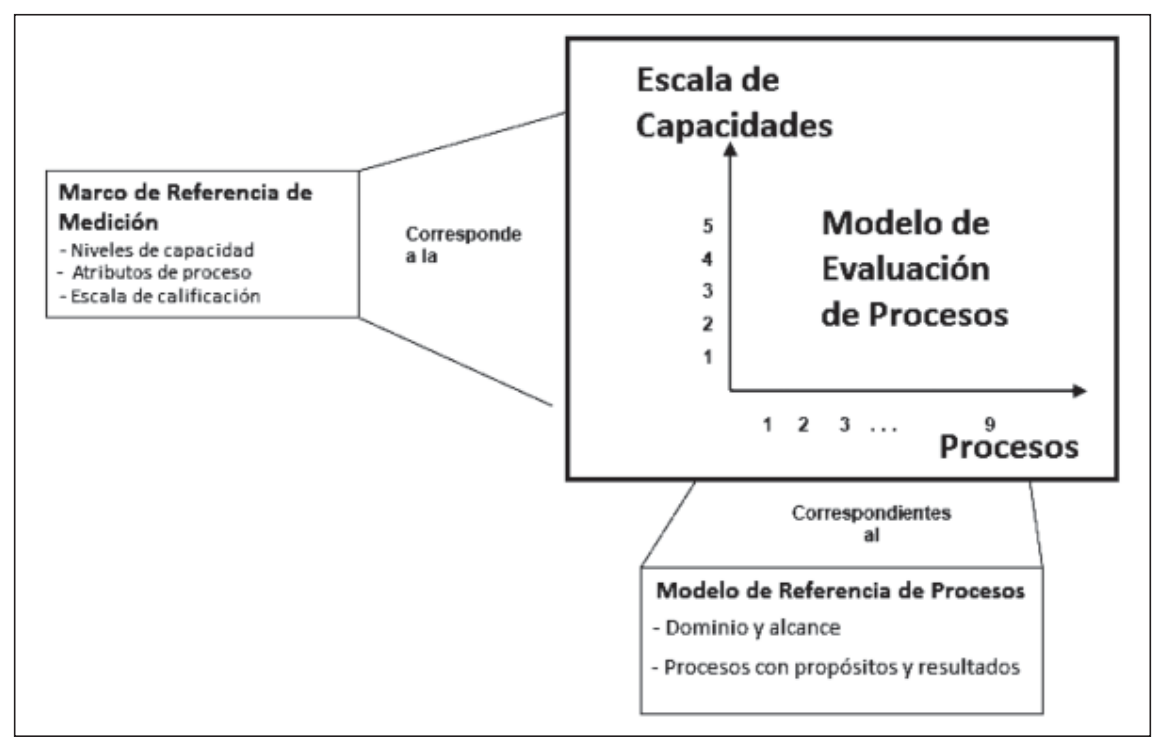

Fuente: Adaptado de ISO/IEC 15504-2 [ISO 2003].

Figura 4. Elementos del método de evaluación tomado de ISO/IEC 15504-2 [ISO 2003].

La norma define para cada nivel de capacidad un conjunto de atributos que deben ser satisfechos, cada atributo tiene un conjunto de hechos que permiten identificar su logro [ISO 2003] y posteriormente, según [ISO 2004b], en cada hecho se pueden identificar elementos que se pueden evaluar de manera directa, para ver si se cumple o no. A manera de ejemplo, en la Tabla 1 se presenta el nivel de capacidad y el estado que le corresponde, los atributos de cada estado de proceso, las condiciones que determinan el logro del atributo y los elementos a considerar solamente de los niveles 1 y 2 . 
Tabla 1. Extracto de niveles de capacidad y elementos para su evaluación, obtenido de ISO/IEC 15504 parte 2 y parte 3 .

\begin{tabular}{|c|c|c|c|c|}
\hline Nivel & Estado & Atributo de proceso & Condición o hecho & Elementos a considerar \\
\hline 0 & Incompleto & Ninguno & Ninguno & Ninguno \\
\hline 1 & Realizado & $\begin{array}{l}\text { AP 1.1. Atributo de } \\
\text { realización del proceso }\end{array}$ & $\begin{array}{l}\text { a. El proceso obtiene los resulta- } \\
\text { dos definidos. }\end{array}$ & $\begin{array}{l}\text { Se cumplen con las Entradas. Se cumple con las Salidas. Se } \\
\text { realizan las acciones para convertir entradas en salidas. }\end{array}$ \\
\hline \multirow{10}{*}{2} & \multirow{10}{*}{ Administrado } & \multirow{6}{*}{$\begin{array}{l}\text { AP 2.1. Atributo de } \\
\text { administración de la } \\
\text { realización }\end{array}$} & $\begin{array}{l}\text { a. Los objetivos de desempeño } \\
\text { del proceso están definidos. }\end{array}$ & $\begin{array}{l}\text { Se define la calidad de los artefactos producidos. Se define } \\
\text { el tiempo del ciclo de procesos. Se define la utilización de re- } \\
\text { cursos. }\end{array}$ \\
\hline & & & $\begin{array}{l}\text { b. El desempeño del proceso } \\
\text { está planeado y monitoreado. }\end{array}$ & $\begin{array}{l}\text { Se tiene el plan de trabajo. Se utiliza el plan de trabajo. Se } \\
\text { emite el reporte de avances. Se revisa el reporte de avance. }\end{array}$ \\
\hline & & & $\begin{array}{l}\text { c. El desempeño del proceso } \\
\text { está ajustado de acuerdo a lo } \\
\text { planeado. }\end{array}$ & $\begin{array}{l}\text { Se maneja el control de cambios de planes. Se actualiza los } \\
\text { planes de trabajo. }\end{array}$ \\
\hline & & & $\begin{array}{l}\text { d. Las responsabilidades y auto- } \\
\text { ridades para el desempeño del } \\
\text { proceso están definidas, asigna- } \\
\text { das y comunicadas. }\end{array}$ & $\begin{array}{l}\text { Se definen los responsables y sus niveles de autoridad. Se } \\
\text { asignan las responsabilidades. Se comunican las responsabi- } \\
\text { lidades y nivel de autoridad. }\end{array}$ \\
\hline & & & $\begin{array}{l}\text { e. Están identificadas, dispo- } \\
\text { nibles, asignados y utilizados } \\
\text { los recursos e información ne- } \\
\text { cesaria para el desempeño del } \\
\text { proceso. }\end{array}$ & $\begin{array}{l}\text { Se identifican los recursos. Se dispone de los recursos según } \\
\text { lo previsto. Se utiliza los recursos. Se identifica la información. } \\
\text { Se dispone de información según lo previsto. Se utiliza la in- } \\
\text { formación. }\end{array}$ \\
\hline & & & $\begin{array}{l}\text { f. Las interfaces entre las par- } \\
\text { tes involucradas están admi- } \\
\text { nistradas para asegurar una } \\
\text { comunicación efectiva y para la } \\
\text { asignación clara de las respon- } \\
\text { sabilidades. }\end{array}$ & $\begin{array}{l}\text { Se comunican de manera efectiva los involucrados. Se tiene } \\
\text { claridad en la asignación de las responsabilidades. }\end{array}$ \\
\hline & & \multirow{4}{*}{$\begin{array}{l}\text { AP 2.2. Atributo de } \\
\text { administración de los } \\
\text { entregables }\end{array}$} & $\begin{array}{l}\text { a. Los requerimientos para los } \\
\text { entregables del proceso están } \\
\text { definidos. }\end{array}$ & $\begin{array}{l}\text { Se definen los requerimientos para las entregables del pro- } \\
\text { ducto. Se definen los requerimientos de los entregables de } \\
\text { la gestión. }\end{array}$ \\
\hline & & & $\begin{array}{l}\text { b. Los requerimientos para la } \\
\text { documentación y control de los } \\
\text { entregables están definidos. }\end{array}$ & $\begin{array}{l}\text { Se definen los requerimientos de documentación de los en- } \\
\text { tregables. Se defienen los requerimientos de control de los } \\
\text { entregables. }\end{array}$ \\
\hline & & & $\begin{array}{l}\text { c. Los entregables están apro- } \\
\text { piadamente identificados, docu- } \\
\text { mentados y controlados. }\end{array}$ & $\begin{array}{l}\text { Se identifican de manera apropiada los entregables. Se docu- } \\
\text { mentan de manera apropiada de los entregables. Se controlan } \\
\text { de manera adecuada los entregables. }\end{array}$ \\
\hline & & & $\begin{array}{l}\text { d. Los entregables están revi- } \\
\text { sados en concordancia con los } \\
\text { planes y son ajustados si es } \\
\text { necesario en base a los reque- } \\
\text { rimientos. }\end{array}$ & $\begin{array}{l}\text { Se revisan los entregables de acuerdo a los planes. Se ajus- } \\
\text { tan los entregables de acuerdo a los requirimientos. }\end{array}$ \\
\hline
\end{tabular}

Fuente: Adaptado de ISO/IEC 15504 partes 2 y 3. [ISO 2003],[ISO 2004b]

\section{CÁLCULO DE LA CAPACIDAD DE LOS PROCE- SOS}

En esta sección se describe brevemente el mapeo de procesos realizado entre MoProSoft y RUP que constituye la base para calcular las capacidades de los procesos, luego se describe el esquema de cálculo utilizado para la determinación de la capacidad de procesos y luego se presentan los resultados obtenidos con este proceso de cálculo.

\section{Mapeo entre MoProSoft y RUP}

Dentro del Proyecto COMPETISOFT, se realizó el mapeo de procesos entre Rational Unified Process (RUP) y los procesos de la categoría de operación de MoProSoft [Canepa 2008]. Este trabajo permitió determinar que tanto los elementos de proceso (actividades, entradas, salidas, etc.) de un modelo está incluido en otro y se llevó a cabo bajo el supuesto que RUP está implementado con 
un conjunto mínimo de elementos que satisfacen la relación con la categoría de Operación de MoProSoft.

En este trabajo se analizó la correspondencia entre distintos elementos de ambos modelos. Esta correspondencia no siempre implica una relación uno a uno entre ellos, ni implica que sea completa. En algunos casos, el contenido de un elemento de MoProSoft ha sido desdoblado hasta en 4 niveles para poder realizar un adecuado análisis de los elementos y poder determinar la adecuada cobertura entre los elementos.

Además, se establecieron reglas que permitieron determinar: por un lado, la multiplicidad entre elementos (dado que uno o muchos elementos de RUP no necesariamente cubren totalmente a un elemento de MoProSoft), y por otro lado, tal como se muestra en la Tabla 2, las reglas de cubrimiento muestran las puntuaciones asignadas a la cobertura alcanzada por los elementos basadas en ISO/ IEC 15504-2 [ISO 2003]. Se debe señalar que la ISO/IEC 15504 define para las calificaciones [N] No cumple, [P] Parcialmente, [A] Ampliamente y [T] Totalmente y no una calificación numérica; siendo los datos de rangos en porcentaje, proporcionados en la Tabla 2, referenciales para dar una idea de las magnitudes de cumplimiento que corresponde con cada calificación de N, P, A y T. De manera correspondiente y a manera de ejercicio académico, se fijó unos valores (puntaje asignado a cada calificación) para poder determinar un valor (numérico) de la cobertura y niveles de capacidad que se determinarán en este trabajo.

Tabla 2. Reglas de cubrimiento

\begin{tabular}{|c|l|r|c|}
\hline Regla & Cobertura & Porcentaje & Puntaje asignado \\
\hline 1 & Totalmente & $85 \%$ a $100 \%$ & 1.0 \\
2 & Ampliamiente & $50 \%$ a $85 \%$ & 0.7 \\
3 & Parcialmente & $15 \%$ a $50 \%$ & 0.3 \\
4 & No cubre & $0 \%$ a $15 \%$ & 0.0 \\
\hline
\end{tabular}

Fuente: Tomado de [Canepa 2008].

A través de estas reglas, se realizó el cálculo de la puntuación del mapeo tomando en cuenta el desdoblamiento de elementos hasta en cuatro niveles. Se determinó el valor de cobertura de cada subdivisión dependiendo del puntaje de cobertura alcanzado por elemento de RUP y el máximo valor a alcanzar en la subdivisión. En la Tabla 3, se muestra un ejemplo del método de cálculo empleado.

Tabla 3. Ejemplo de Método de Cálculo de la Puntuación [Canepa 2008].

\begin{tabular}{|l|c|c|c|c|c|c|c|c|c|c|}
\hline \multicolumn{5}{|c|}{ MoProSoft } & \multicolumn{5}{c|}{ Rational Unified Process } \\
\hline Elemento & $\mathrm{n} 4$ & $\mathrm{n} 3$ & $\mathrm{n} 2$ & $\mathrm{n} 1$ & $\mathrm{n} 1$ & $\mathrm{n} 2$ & $\mathrm{n} 3$ & $\mathrm{n} 4$ & $\mathrm{P}$ & Elemento \\
\hline $\mathrm{A} 1$ & & & & 1 & 0.48 & & & & & \\
\hline A1.1 & & & 0.5 & & & 0.18 & & & & \\
\hline A1.1.1 & & 0.25 & & & & & 0.075 & & 0.3 & $\mathrm{~B} 1.1 .2$ \\
\hline A1.1.2 & & 0.25 & & & & & 0.125 & & & \\
\hline A1.1.2.1 & 0.125 & & & & & & & 0.125 & 1 & B1.1.2.1 \\
\hline A1.1.2.2 & 0.125 & & & & & & & 0 & 0 & \\
\hline A1.2 & & & 0.5 & & & 0.3 & & & 0.6 & B1.2 \\
\hline
\end{tabular}

nx: nivel $\mathrm{x}$

P: Puntaje de cobertura asignado según Tabla 2

Fuente: Tomado de Canepa (2008).

Se determinó que el cubrimiento de RUP respecto a MoProSoft se da en alrededor de un $90 \%$. Con un $84 \%$ para el proceso de Administración de Proyectos Específicos y con $95 \%$ para el proceso de
Desarrollo y Mantenimiento de Software. La Tabla 4 , muestra los resultados generales que se obtuvieron del mapeo. 
Tabla 4. Resultados de la Cobertura de RUP respecto a MoProSoft [Canepa 2008].

\begin{tabular}{|l|c|c|c|}
\hline \multicolumn{1}{|c|}{$\begin{array}{c}\text { Total - Categoría } \\
\text { Operación }\end{array}$} & \multicolumn{3}{c|}{ Cobertura MoProSoft a RUP } \\
\hline Elementos & $\begin{array}{c}\text { Elementos de } \\
\text { MoProSoft }\end{array}$ & $\begin{array}{c}\text { Elementos } \\
\text { de RUP }\end{array}$ & $\begin{array}{c}\text { Porcentaje } \\
\text { cubierto por } \\
\text { RUP (\%) }\end{array}$ \\
\hline Entradas & 13.0 & 11.2 & 86.2 \\
\hline Salidas & 23.0 & 19.7 & 85.7 \\
\hline Productos intermedios & 6.0 & 6.0 & 100.0 \\
\hline Roles involucrados y capacitación & 18.0 & 18.0 & 100.0 \\
\hline Actividades & 88.0 & 79.0 & 89.7 \\
\hline Total de la categoría & 148.0 & 133.9 & 90.5 \\
\hline
\end{tabular}

Fuente: Tomado de Canepa (2008)

\section{Descripción del método de cálculo}

Existe una versión de MoProSoft coloreada que representan los distintos niveles de madurez. Cada color asignado representa un nivel de madurez que está en correspondencia con lo definido en la norma internacional ISO/IEC 15504 [ISO 2003][Oktaba 2005]. Para determinar el nivel de capacidad de los procesos de RUP respecto a MoProSoft se utilizó esta versión coloreada en donde se presenta al modelo MoProSoft y todos sus elementos, resaltados con el color asociado al nivel de capacidad que corresponden (del nivel 1 al nivel 5)

El proceso seguido consistió en: (i) la identificación de los elementos de RUP asociados al mapeo que pertenecen a cada uno de los niveles de MoProSoft (niveles del 1 al 5), (ii) la determinación del nivel alcanzado sobre el mapeo realizado previamente, que se obtuvo a partir de la cobertura alcanzada de los elementos de RUP hacia MoProSoft en cada uno de estos niveles, contando los elementos que pertenecen a cada nivel, y (iii) el cálculo del porcentaje de cubrimiento. Finalmente, se repitió el proceso para cada uno de los 5 niveles y procesos de MoProSoft.

\section{Resultados}

En base al método del cálculo descrito en el punto 4.2 , se obtuvieron los resultados que permiten determinar el nivel de capacidad alcanzado por el conjunto de elementos de RUP respecto a los procesos de la categoría de operación de MoProSoft. En la Tabla 5, se muestran dichos resultados en función a cada nivel y a los dos procesos de la Categoría de Operación (Proceso de Administración de Proyecto Específicos y el Proceso de Desarrollo y Mantenimiento de Software).

Las columnas LM de la Tabla 5 representan los límites máximos que puede ser alcanzado; para este trabajo, estos valores están determinados por el total de elementos de MoProSoft considerados en el nivel y el proceso analizado. Las columnas AR representan los niveles alcanzados por RUP, para ello se ha considerado que los elementos de RUP pueden cubrir parcialmente los elementos de MoProSoft, tal como se completo en el mapeo [Canepa 2008], por lo que el total (sumatoria) es un valor con decimales. Por ejemplo, en el nivel 1 del proceso de Administración de Proyectos Específicos (PAPE) se observa que el límite máximo es 25. Esto quiere decir que existen 25 elementos de este proceso en el nivel 1. Para este mismo nivel, la cobertura alcanzada por RUP es de 23.2 elementos, es decir, RUP cubre $92.6 \%$ a MoProSoft en el nivel 1 de APE.

La Tabla 5 muestra el porcentaje alcanzado en cada uno de los niveles de los dos procesos y el total en función a la cantidad de elementos y al porcentaje cubierto por RUP de los dos procesos de MoProSoft. Como ejemplo podemos observar que en el nivel 1, RUP cubre el $93 \%$ de los elementos de MoProSoft en el proceso de Administración de Proyectos Específicos; $100 \%$ en el proceso de Desarrollo y Mantenimiento de Software. Finalmente se observa que se alcanza el $97 \%$ en el nivel 1.

Tabla 5. Resultados de la cobertura alcanzada en cada nivel.

\begin{tabular}{|l|c|c|c|c|c|c|c|c|c|c|}
\cline { 2 - 12 } \multicolumn{1}{c|}{} & \multicolumn{2}{c|}{ NIVEL 1 } & \multicolumn{2}{c|}{ NIVEL 2 } & \multicolumn{2}{c|}{ NIVEL 3 } & \multicolumn{2}{c|}{ NIVEL 4 } & \multicolumn{2}{c|}{ NIVEL 5 } \\
\cline { 2 - 12 } \multicolumn{1}{c|}{} & LM & AR & LM & AR & LM & AR & LM & AR & LM & AR \\
\hline PAPE & 25.0 & 23.2 & 29.2 & 24.3 & 6.5 & 4.7 & 4.3 & 2.3 & 0.0 & 0.0 \\
\hline PAPE \% & & 92.6 & & 83.4 & & 72.3 & & 54.0 & & 0.0 \\
\hline PDMS & 29.4 & 29.4 & 46.8 & 45.0 & 4.3 & 2.3 & 2.5 & 2.5 & 0.0 & 0.0 \\
\hline PDMS \% & & 100.0 & & 96.2 & & 53.6 & & 100.0 & & 0.0 \\
\hline TOTAL & 54.4 & 52.6 & 76.0 & 69.4 & 10.8 & 7.0 & 6.8 & 4.8 & 0.0 & 0.0 \\
\hline \%TOTAL & & $\mathbf{9 6 . 6}$ & & $\mathbf{9 1 . 3}$ & & $\mathbf{6 4 . 9}$ & & $\mathbf{7 0 . 9}$ & & $\mathbf{0 . 0}$ \\
\hline
\end{tabular}

LM: Límite MoProSoft

AR: Alcanzado por RUP.

Fuente: Elaboración propia.

\section{DISCUSIÓN DE RESULTADOS}

En este trabajo se analizó la capacidad alcanzada por el Proceso de Administración de Proyecto Específico (PAPE) y el Proceso de Desarrollo y Mantenimiento de Software (PDMS), ambos de la categoría de operación de MoProSoft bajo el supuesto que la empresa tiene implementado de manera adecuada un conjunto elementos y esquema de trabajo basado de RUP; estos elementos fueron determinados en un trabajo previo [Canepa, 2008]. Para el análisis se ha utilizado el modelo coloreado de MoProSoft que incorpora los niveles a través de asignación de colores a los distintos elementos del mismo. 
Para el nivel 1, según los resultados obtenidos al evaluar el nivel de capacidad de los procesos del mapeo, se observa que ambos procesos logran para este nivel, la calificación de Completamente Alcanzado según ISO/IEC 15504. Para el caso de PAPE se tiene que el LM es 25.0 y el AR es 23.2, lo que da una puntuación de $92.6 \%$ del nivel 1 . Situación semejante ocurre con el PDMS, donde LM es 29.4 y el AR es 29.4, lo que da un puntuación del $100 \%$ del nivel 1 . Este resultado no escapa a lo previsible teniendo en cuenta que RUP principalmente se ocupa de la parte de técnica del desarrollo de software.

En el nivel 2, según las ISO/IEC 15504, para el caso de PAPE se obtiene que la calificación (83.4\%) es de Ampliamente Alcanzado, y para el caso de PDMS la calificación es de Completamente Alcanzado pues la calificación es de $96.2 \%$. En este caso se nota con mayor claridad que RUP tiene un mayor énfasis en el lado técnico.

Para los niveles 3 y 4 se presentan una situación particular: (i) según el modelo MoProSoft, se tiene una pequeña cantidad de elementos con respecto a los cuales se hace la medición, (ii) en el caso de PAPE se nota un claro descenso de la puntuación lo que es correspondiente con el menor énfasis que tiene este aspecto dentro de RUP, (iii) en el caso de PDMS ocurre una descenso y luego crecimiento en la calificación; lo que se puede explicar y aceptar por la poca cantidad de elementos que se tienen en esos dos niveles. Para el nivel 5 no se presentan elementos en el modelo MoProSoft por lo que no ha sido posible hacer dicha evaluación.

En la Tabla 6 se muestra un resumen de las calificaciones obtenidas en cada proceso, basado en ISO/ IEC 15504. Se observa que el proceso de administración de proyectos específicos alcanza el nivel 2 de capacidad y el proceso de desarrollo y mantenimiento de software el nivel 3.

Tabla 6. Calificaciones alcanzadas por cada nivel.

\begin{tabular}{|l|c|c|c|c|c|}
\cline { 2 - 6 } \multicolumn{1}{c|}{} & NIVEL 1 & NIVEL 2 & NIVEL 3 & NIVEL 4 & NIVEL 5 \\
\hline PAPE & $\mathrm{T}$ & $\mathrm{A}$ & $\mathrm{A}$ & $\mathrm{A}$ & - \\
\hline PDMS & $\mathrm{T}$ & $\mathrm{T}$ & $\mathrm{A}$ & $\mathrm{T}$ & - \\
\hline
\end{tabular}

Fuente: Elaboración propia.

\section{CONCLUSIÓN Y TRABAJO FUTURO}

En base al análisis realizado se puede afirmar que efectivamente, RUP cubre varias prácticas de los niveles de capacidad de MoProSoft, que para el supuesto que una empresa tenga implementado de manera adecuada un esquema de trabajo basado en un conjunto de elementos de RUP, determinadas previamente [Canepa 2008], tendría muy probablemente una capacidad de nivel 3 en el procesos de Desarrollo y Mantenimiento de Software (PDMS) y capacidad de nivel 2 en el proceso de Administración de Proyecto Específico (PAPE) de MoProSoft. Para el caso de una organización real se debe tener en cuenta que la evaluación se hace en función a evidencias que demuestren que los artefactos y actividades se cumplen, lo que puede no ser cierto en algunos casos y por tanto los valores calculados de manera teórica no ser necesariamente alcanzables.

Como trabajo futuro se tiene previsto la elaboración de una guía que oriente a las empresas desarrolladoras de software que tienen implementado RUP a identificar aquellos otros elementos mínimos necesarios de RUP para que puedan implementar y luego ser evaluados bajo el modelo MoProSoft. Asimismo, se revisará la posibilidad de hacer un trabajo semejante con otros modelos y marcos de referencia.

\section{RECONOCIMIENTOS}

El presente trabajo está enmarcado dentro del proyecto 506AC0287 COMPETISOFT (Mejora de Procesos Para Fomentar la Competitividad de la Pequeña y Mediana Industria de Software de Iberoamérica) del programa CYTED (Ciencia y Tecnología para el Desarrollo) y apoyado parcialmente por la Dirección de Gestión de Investigación (Proyecto DGI-PUCP-008-2009) y el Departamento de Ingeniería de la Pontificia Universidad Católica del Perú.

\section{REFERENCIAS BIBLIOGRÁFICAS}

[1] Astorga, M., Olguín, J., y Flores, B., (2006). "Caracterización de los factores de cambio para la implantación de MoProSoft en las pymes". En: Actas del 1. er Congreso Internacional de Ciencias de la Computación. CiComp 06. Pp. 13-17. Baja California, México.

[2] Canepa, K., y Dávila, A., (2008). "Mapeo de los Procesos de RUP respecto a MoProSoft". En: Actas del VII Jornadas Iberoamericanas de Ingeniería de Software e Ingeniería del Conocimiento. Pp. 139-146. Guayaquil, Ecuador.

[3] COMPETISOFT (2006). Proyecto de mejora de procesos para fomentar la competitividad de la pequeña y mediana industria del software de Iberoamérica. http://alarcos.inf-cr.uclm.es/ Competisoft/ 
[4] Dávila, A (2009). "NTP 291.100 norma técnica para pymes que desarrollan software". En el evento Presentación Oficial de la NTP 291.100 - MOPROSOFT. http://www.acklis.com/recursos/eventos.html

[5] Heinz, L. (2003). "CMMI Adoption Trends". In: new@sei, Software Engineering Institute. Carnegie Mellon University. http://www.sei.cmu. edu/news-at-sei/features/2003/4q03/feature1-4q03.htm.

[6] IBM (2007). Rational Unified Process, IBM Rational Method Componer, V. 7.1.1.

[7] ISO (2003). Standard ISO/IEC 15504-2:2003. Information Technology - Process assessment - Part 2 Performing an assessment. Ginebra, Suiza.

[8] ISO (2004). Standard ISO/IEC 90003:2004. Software engineering - Guidelines for the application of ISO 9001:2000 to computer software. Ginebra, Suiza.

[9] ISO (2004a). Standard ISO/IEC 12207:2004 Information Technology - Software life cycle processes. Ginebra, Suiza.

[10] ISO (2004b). Standard ISO/IEC 15504-3:2004. Information Technology - Process assessment Part 3 Guidance on performing an assessment. Ginebra, Suiza.

[11] ISO (2004c). Standard ISO/IEC 15504-1:2004. Information Technology - Process assessment - Part 1: Concepts and Vocabulary. Ginebra, Suiza.

[12] Jacobson, I., Booch, G., and Rumbaugh, J., (1999). Unified Software Development Process, Addison-Wesley.

[13] Kruchten, P. (2003). The Rational Unified Process: An Introduction, Third Edition, Addison Wesley.

[14] Laporte, C., (2008). ISO/IEC JTC1/SC7 Working Group 24 Home Page. http://profs. logti.etsmtl.ca/claporte/English/VSE/index. html.
[15] Mogrovejo, J., y Dávila, A., (2008). "Una Experiencia de Implantación de COMPETISOFT en una Pequeña Empresa Desarrolladora de Software". En: Actas del VII Jornadas Iberoamericanas de Ingeniería de Software e Ingeniería del Conocimiento. Pp. 67-71. Guayaquil, Ecuador

[16] Oktaba H., et al. (2005). Modelo de Procesos para la Industria de Software, MoProSoft. Versión 1.3, en Colores del Modelo de Procesos de Software. http://www.software. net.mx/desarrolladores/prosoft/temas_interes/ moprosoft.htm

[17] Oktaba, H.: (2005a). "MoProSoft: A Software Process Model for Small Enterprises". In: Proceedings of the First International Research Workshop for Process Improvement in Small Settings, pp. 93-100. Software Engineer Institute, Carnegie Mellon University.

[18] Oktaba, H., Piattini, M., García, F., Pino, F. Alquicira, C., y Ruiz, F., (2007). "Software Process Improvement: The COMPETISOFT Project". In: IEEE Computer. Vol. 40, N.10. October 2007. p: 21-28.

[19] Rational (1998), Rational Unified Process., Best Practices for Software Development Teams, Rational Software White Paper, TP026B, Rev $11 / 01$.

[20] Sánchez, G., Vergara, D., y Dávila, A., (2008) "Experiencia de Implementación de Mejora de Procesos en dos PYMEs Desarrolladoras de Software, que poseen certificación ISO 9001:2000". En: Actas del VII Jornadas Iberoamericanas de Ingeniería de Software e Ingeniería del Conocimiento. Pp. 73-80. Guayaquil, Ecuador.

[21] SOFTEX (2007). Melhoria de Processos do Software. Associação para Promoção da Excelência do Software Brasileiro - SOFTEX. Campinas. Brasil. http://www.softex.br/mpsbr/ home/default.asp 